\title{
Resveratrol protects against sodium nitroprusside induced nucleus pulposus cell apoptosis by scavenging ROS
}

\author{
$\mathrm{KANG} \mathrm{LI}^{1 *}, \mathrm{YAN} \mathrm{LI}^{1 *}, \mathrm{JIE} \mathrm{MI}^{1}, \mathrm{LU}^{*} \mathrm{MAO}^{2}, \mathrm{XIUGUO} \mathrm{HAN}^{1}$ and $\mathrm{JIE} \mathrm{ZHAO}^{1}$ \\ ${ }^{1}$ Shanghai Key Laboratory of Orthopaedic Implants, Department of Orthopaedics, Shanghai Ninth People's Hospital, \\ Shanghai Jiao Tong University School of Medicine, Shanghai 200011; ${ }^{2}$ Spine Center, Zhongda Hospital, \\ Southeast University, Nanjing, Jiangsu 210009, P.R. China
}

Received January 16, 2016; Accepted January 10, 2018

DOI: $10.3892 /$ ijmm.2018.3461

\begin{abstract}
Oxidative stress induced disc cell apoptosis plays an important role in intervertebral disc (IVD) degeneration. The present study aims to investigate effects of resveratrol (RV), a natural polyphenol compound, on sodium nitroprusside (SNP) induced nucleus pulposus (NP) cell apoptosis and related mechanism. Rat NP cells were pretreated with RV, N-acetyl cysteine (NAC) and carboxy-PTIO (PTIO) before SNP treatment. Cell Counting Kit-8 assay was carried out for cell viability evaluation. Annexin V/propidium iodide (PI), Hoechst 33258 and Actin-Tracker Green and Tubulin-Tracker Red staining were conducted to detect NP cell apoptosis and apoptotic structural changes. Mitochondrial membrane potential $(\Delta \Psi \mathrm{m})$ was analyzed with tetramethylrhodamine methyl ester staining. DCFH-DA and DAF-FM DA staining was used to determine intracellular reactive oxygen species (ROS) and nitric oxide (NO) levels. An ex vivo experiment was also carried out followed by TUNEL assay of sections of discs. SNP induced NP cell apoptosis, excessive production of intracellular ROS and NO, reduction of $\Delta \Psi \mathrm{m}$ as well as disruption of cytoskeletal and morphological structure. Meanwhile, organ culture results showed that SNP induced NP cell apoptosis ex vivo. RV and NAC siginificantly inhibited SNP induced NP cell apoptosis, production of intracellular ROS, deline of $\Delta \Psi_{\mathrm{m}}$ as well as disruption of cytoskeletal and morphological structure, while RV did not suppress NO production. RV and NAC could also suppress SNP induced NP cell apoptosis ex vivo. However, PTIO did not prevent SNP induced NP cell apoptosis, though it scavenged NO significantly. In conclusion, RV
\end{abstract}

Correspondence to: Professor Jie Zhao, Shanghai Key Laboratory of Orthopaedic Implant, Department of Orthopaedics, Shanghai Ninth People's Hospital, Shanghai Jiao Tong University School of Medicine, 639 Zhizaoju Road, Shanghai 200011, P.R. China

E-mail: spinezhaojie@163.com

${ }^{*}$ Contributed equally

Key words: nucleus pulposus, apoptosis, resveratrol, reactive oxygen species, organ culture protects against SNP induced NP cell apoptosis by scavenging ROS but not NO, suggesting a promising prospect of RV in IVD degeneration retardation.

\section{Introduction}

Low back pain (LBP), a serious social health problem in modern society, has imposed a huge burden on the health care system $(1,2)$. Related studies have proved intervertebral disc (IVD) degeneration to play the most important role in pathology of LBP and other spine degenerative diseases $(3,4)$. Nowadays, however, the IVD degeneration mechanism has not be completely elucidated. There is evidence that nucleus pulposus (NP) cell apoptosis, which is triggered by biomechanical and biochemical stimulus in degenerative progression $(5,6)$, plays an important role in IVD degeneration $(7,8)$.

Previous studies demonstrate that excessive reactive oxygen species (ROS) may impair the mitochondrial function, resulting in apoptosis (9). In IVD, ROS can be produced by NP cells in response to many kinds of biomechanical and biochemical stimulus, such as compression loading, high glucose and hydrogen peroxide (10-13). Meanwhile, nitric oxide (NO) is also proved to induce IVD cell apoptosis during degeneration (14). NO can suppress activity of cytochrome oxidase, leading to the reduction of the electron transport chain and the production of superoxide anions (15). NO production of NP cells may be induced by stimulation of interleukin (IL)-1, -10 and interferon (INF) $-\gamma$, which are involved in IVD degeneration $(16,17)$. Sodium nitroprusside (SNP), a widely used donor of NO, is often adopted to investigate the mechanism of chondrocyte apoptosis induced by NO (18-21). Treatment of SNP can also cause mitochondrial dysfunction in chondrocyte, which is characterized by decline of mitochondrial membrane potential $\left(\Delta \Psi_{\mathrm{m}}\right)$ and release of cytochrome $c$ (21-23). Similarly, as to IVD, SNP has been also used as an apoptosis inducer in annulus fibrosus cells, which are chondrocyte-like, suggesting that SNP can induce both endoplasmic reticulum and mitochondrial stress in annulus fibrosus cells (24).

Resveratrol (RV; 3,5,4'-trihydroxy-trans-stilbene), a natural polyphenol compound which could be extracted from grapes, has previously been proved to possess anti-inflammatory, 
antioxidant, and anticancer bioactivities in different kinds of cells and tissues (25-29). RV can protect IVD from degeneration by reducing levels of proinflammatory cytokines and activating silent mating type information regulator 2 homolog 1 (SIRT1) (30-34). However, antioxidant properties of RV in IVD have not been elucidated yet.

The present study aimed to explore the effects of RV on SNP induced NP cell apoptosis and investigate related mechanism. For the first time, our results indicate that $\mathrm{RV}$ can protect NP cells from SNP induced apoptosis, through scavenging excessive ROS rather than NO provided by SNP.

\section{Materials and methods}

Materials and reagents. SNP, RV, dimethyl sulfoxide (DMSO), $\mathrm{N}$-acetyl cysteine (NAC), carboxy-PTIO (PTIO), type II collagenase, L-ascorbic acid, Hoechst 33258, 4',6-diamidino-2-phenylindole (DAPI) and insulin-transferrin-selenium were purchased from Sigma-Aldrich (St. Louis, MO, USA). Dulbecco's modified Eagle's medium/nutrient mixture F-12 (DMEM/F12), trypsin, penicillin/streptomycin and fetal bovine serum (FBS) was purchased from Gibco (Carlsbad, CA, USA). Cell Counting Kit-8 (CCK-8) was purchased from Dojindo (Kumamoto, Japan). Caspase-3, -8, and -9 activity assay kits, 2',7'-dichlorofluorescin diacetate (DCFH-DA), 3-amino,4-aminomethyl-2',7'-difluorescein, diacetate (DAF-FM DA), Actin-Tracker Green and Tubulin-Trakcer Red were from purchased from Beyotime Institute of Biotechnology (Jiangsu, China). Annexin V-FITC/propidium iodide (PI) apoptosis detection kit and tetramethylrhodamine methyl ester (TMRM) was purchased from Invitrogen Life Technologies (Carlsbad, CA, USA). In situ cell death detection kit was purchased from Roche Diagnostics (Basel, Switzerland).

SNP was dissolved with phosphate-buffered saline (PBS) and NAC was dissolved with ultrapure water just before experiment. RV and PTIO were dissolved with DMSO. It is ensured that the working concentration of DMSO in medium was $<1 \%$ throughout all experiments. The treatment time and concentration of RV and SNP were determined by our experiments. The pretreatment time of PTIO and NAC is $4 \mathrm{~h}$, while their concentration is respectively $100 \mu \mathrm{M}$ and $2 \mathrm{mM}$.

NP cell isolation and culture. This present study was carried out in accordance with the recommendations in the Guide for the Care and Use of Laboratory Animals of the National Institutes of Health. Our experiment protocol was approved by the Animal Care and Experiment Committee of Shanghai Jiao Tong University School of Medicine. Ten 3-month-old Sprague-Dawley rats were provided by Experimental Animal Center of Shanghai Ninth People's Hospital for in vitro experiments. NP cell isolation and culture were carried out as we previously described (35). The second-generation NP cells were adopted in the following experiments. NP cells were seeded into 96 -well plates ( $5 \times 10^{3} /$ well for cell viability assay) or 6 -well plates $\left(1 \times 10^{5} /\right.$ well for apoptosis assay, caspases activity assay, intracellular ROS and NO measurement and mitochondrial membrane potential assay) for at least $24 \mathrm{~h}$ before treatment of any reagent.
Cell viability, apoptosis and caspase activity assay. According to manufacturer's instructions, cell viability was measured with CCK-8 (Dojindo) using a microplate reader (Omega Bio-Tek, Inc., Norcross, GA, USA). All cell viability experiments were performed 6 times. Flow cytometry (FCM) was carried out for analysis of apoptosis rates with Annexin V-FITC/PI apoptosis detection kit following instructions, while 10,000 NP cells were collected for each FCM analysis. Apoptosis rates were calculated as Q2 (Annexin V-FITC-positive and PI-positive) + Q3 (Annexin V-FITC-positive and PI-negative). NP cells were stained with $0.5 \mu \mathrm{g} / \mathrm{ml}$ Hoechst 33258 for $20 \mathrm{~min}$ in dark and then imaged by a fluorescent microscope (IX71; Olmypus, Tokyo, Japan). Caspase-3, -8 and -9 activities were detected with caspase- $-3,-8$, and -9 activity assay kits following manufacturer's instructions using a microplate reader (Omega Bio-Tek, Inc.). The caspase activity levels were expressed as relative activity with control as standard.

Mitochondrial membrane potential assay. Mitochondrial membrane potential was measured with TMRM staining. $\mathrm{NP}$ cells were incubated with $100 \mathrm{nM}$ TMRM at $37^{\circ} \mathrm{C}$ in dark for $30 \mathrm{~min}$, washed with PBS 3 times and covered with fresh medium. NP cells were subsequently imaged using microscope (IX71, Olmypus). The excitation wavelength for TMRM was $549 \mathrm{~nm}$. Three images (x200) of each kind of treatment were obtained for quantitative analysis of fluorescence intensity of the TMRM using IPP version 6.0 software (Media Cybernetics, Bethesda, MD, USA). The fluorescence intensity of the TMRM was expressed as mean density in IPP version 6.0 software.

Measurement of intracellular ROS and NO. To measure intracellular ROS or NO level, NP cells was incubated with DCFH-DA $(10 \mu \mathrm{M})$ for 30 min or with DAF-FM DA $(5 \mu \mathrm{M})$ for $20 \mathrm{~min}$ in dark at $37^{\circ} \mathrm{C}$. After washed with PBS 3 times, NP cells were collected and suspended in fresh medium. The fluorescence intensity was detected using a microplate reader (Omega Bio-Tek, Inc.). The excitation wavelengths for DCFH-DA and DAF-FM DA are 488 and $495 \mathrm{~nm}$ respectively. The experiments were repeated 3 times.

Imaging of cytoskeletal and morphological structure. Imaging of cytoskeletal structure was carried out as previously described (29). After staining with Actin-Tracker Green and Tubulin-Trakcer Red, cytoskeletons (x400) were imaged using a fluorescent microscope (LEICA DM4000B; Leica Microsystems GmbH, Wetzlar, Germany). The excitation wavelengths for Actin-Tracker Green and Tubulin-Trakcer Red are 488 and $543 \mathrm{~nm}$ respectively. After cell treatment, NP cells (x200) were imaged under transmitted light illumination using microscope (IX71; Olmypus).

Organ culture and TUNEL assay. Rat disc organ culture was carried out as we previously described (35). Ex vivo, discs were pretreated with $100 \mu \mathrm{M}$ RV for $24 \mathrm{~h}$ or $2 \mathrm{mM}$ NAC for $12 \mathrm{~h}$ or $100 \mu \mathrm{M}$ PTIO for $12 \mathrm{~h}$, then treated with or without $1 \mathrm{mM}$ SNP for $18 \mathrm{~h}$. Then the harvested discs were fixed in $4 \%$ paraformaldehyde, and then decalcified with EDTA for 2 weeks. After embedding with paraffin, $5-\mu \mathrm{m}$ thick serial mid-sagittal sections of discs were made for slides. Mid-sagittal 

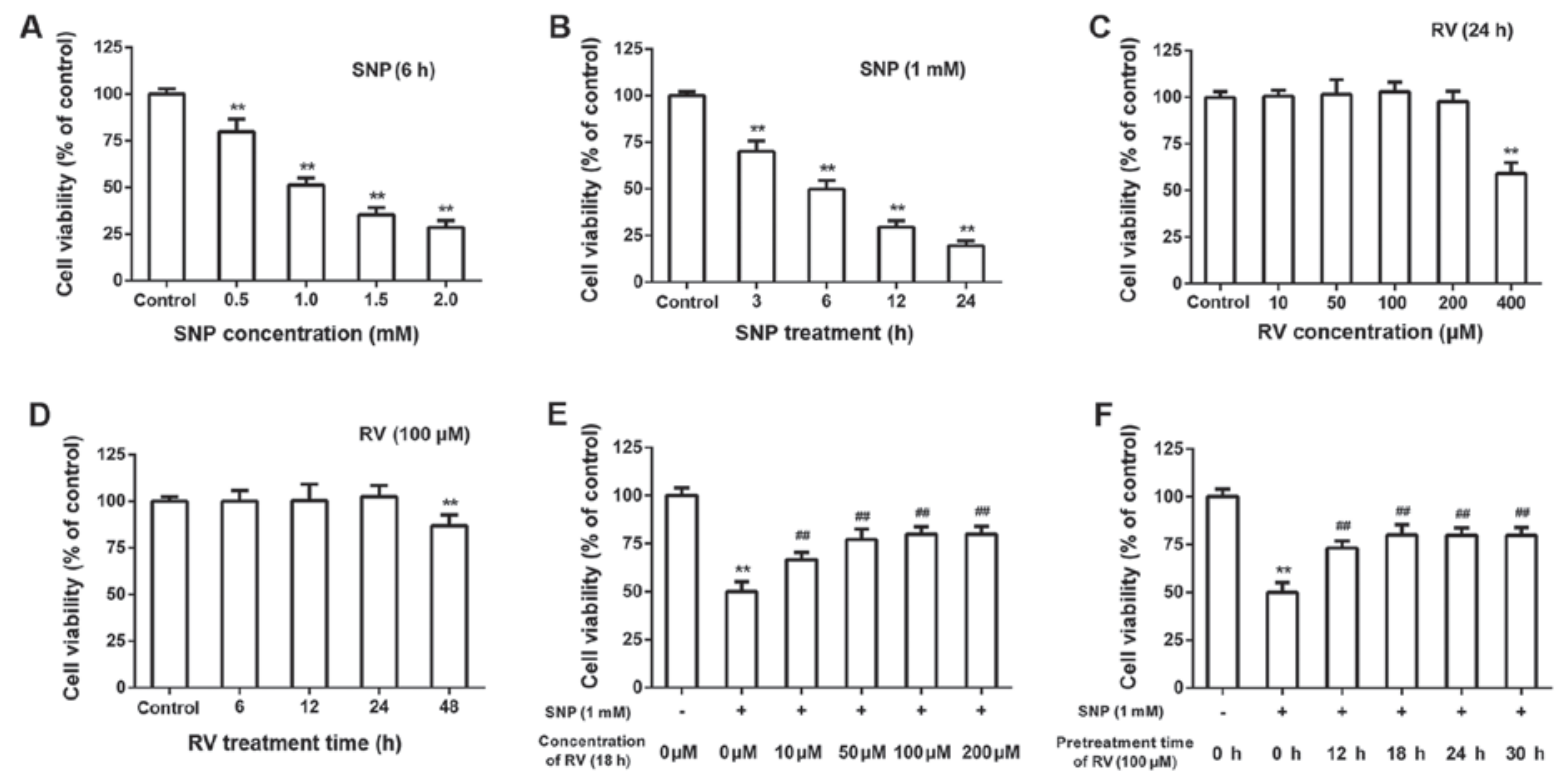

Figure 1. Effects of sodium nitroprusside (SNP) and resveratrol (RV) on nucleus pulposus (NP) cell viability analyzed by Cell Counting Kit-8 (CCK-8) assay. (A and B) Cytotoxicity of SNP on NP cells. (C and D) Cytotoxicity of RV on NP cells. (E and F) Effects of RV on SNP induced NP cell cytotoxiity. Results were expressed as mean \pm standard deviation. ${ }^{* *} \mathrm{p}<0.01$, compared to control; ${ }^{\# \#} \mathrm{p}<0.01$, compared to SNP alone group.

sections of discs were analyzed for apoptosis using in situ cell death detection kit according to manufacturer's instructions. DAPI staining was conducted for indication of total cells. TUNEL-positive apoptotic cells and DAPI-positive total cells of NP area on mid-sagittal sections of discs were identified by fluorescent microscope (IX71; Olmypus), and apoptosis rate was calculated as the percentage of numbers of TUNEL-positive cells to the numbers of total cells using IPP version 6.0 software (Media Cybernetics). The quantitative analysis was performed on three x200 fields/section (three sections/disc and three discs for each kinds of treatment).

Statistical analysis. All statistical data were expressed as mean \pm standard deviation. Results were statistically analyzed by a one-way analysis of variance (ANOVA) with multiple comparisons using SPSS 19.0 (IBM, Inc., New York, NY, USA). P-values $<0.05$ were considered to indicate statistically significant difference.

\section{Results}

Dose and time-dependent effects of SNP and RV on NP cell viability. As shown by CCK- 8 assays, SNP induced NP cells cytotoxicity in a dose and time-dependent manner (Fig. 1A and B). Treatment concentration of SNP was set as $1 \mathrm{mM}$ and treatment time of SNP was set as $6 \mathrm{~h}$ throughout the following experiments without indication. Treatment of RV for $24 \mathrm{~h}$ did not exert obvious cytotoxicity on NP cells when RV concentration was no higher than $200 \mu \mathrm{M}$ (Fig. 1C). Treatment of $100 \mu \mathrm{M}$ RV did not exert significant cytotoxicity on NP cells when treatment time was no longer than $24 \mathrm{~h}$ (Fig. 1D). Pretreatment of RV for $18 \mathrm{~h}$ showed dose-dependent protective effects on SNP induced cytotoxicity (Fig. 1E). Pretreatment of $100 \mu \mathrm{M}$ RV protected NP cells from SNP induced cytotoxicity in a time-dependent manner (Fig. 1F). Treatment concentration of RV was set as
$100 \mu \mathrm{M}$ and treatment time of RV was set as $18 \mathrm{~h}$ throughout the following experiments without indication.

$R V$ protects against SNP induced NP cell apoptosis by scavenging ROS instead of $N O$ in vitro. In order to investigate the mechanism of protective effects of RV, NAC as an established ROS scanvenger and PTIO as an established NO scanvenger were adopted in the following experiments. FCM assay with Annexin V-FITC/PI staining showed that SNP induced significant NP cell apoptosis, which was significantly rescued by RV and NAC instead of PTIO (Fig. 2A and B). CCK-8 assays demonstrated that RV and NAC instead of PTIO significantly suppressed SNP induced cytotoxicity, while NAC or PTIO alone did not show obvious cytotoxicity (Fig. 2C). Meanwhile, RV and NAC instead of PTIO markedly inhibited activation of caspase-3 and -9 induced by SNP (Fig. 2D). However, SNP did not significantly activate caspase-8 (Fig. 2D). Hoechst 33258 staining images showed that RV and NAC instead of PTIO effectively decreased apoptotic nuclei containing condensed or fragmented chromatin induced by SNP, which is consistent with FCM assay with Annexin V-FITC/PI staining (Fig. 2E). As to mitochondrial membrane potential, TMRM staining and quantitative analysis demonstrated that significant loss of $\Delta \Psi \mathrm{m}$ was caused by SNP and effectively rescued by RV and NAC but not PTIO (Fig. 3A and B). As shown by intracellular ROS and NO analysis, SNP significantly induced production of intracellular ROS (Fig. 3C) and NO (Fig. 3D). Pretreatment with RV obviously suppressed SNP induced excessive intracellular ROS production, but did not suppress SNP induced NO production (Fig. 3C and D). As expected, NAC significantly decreased the SNP induced high intracellular ROS and NO production, while PTIO significantly decreased the SNP induced high intracellular NO production but not ROS production.

Overall, ROS, not NO, production is probably the key mechanism of SNP induced NP cell apoptosis. RV can 

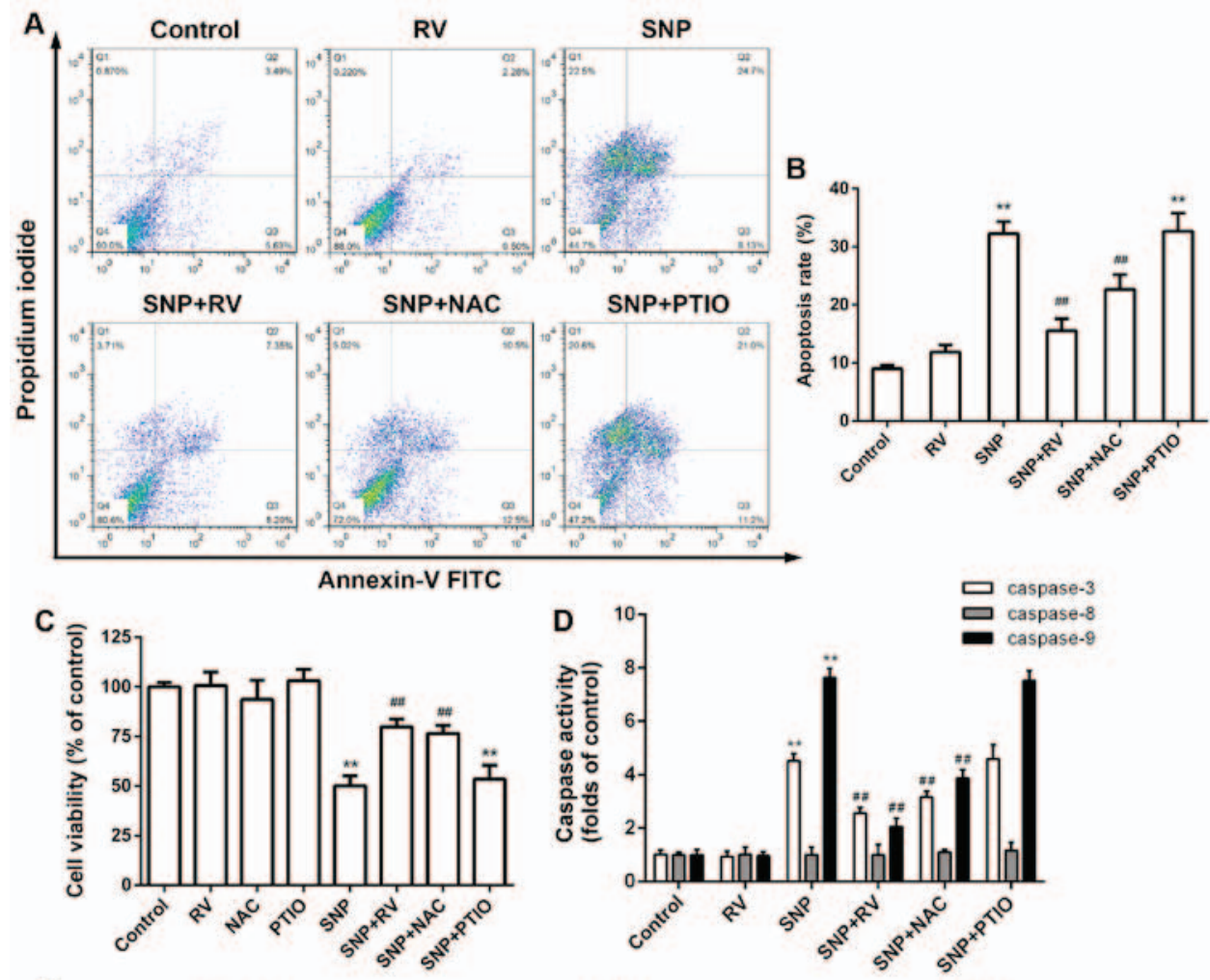

E
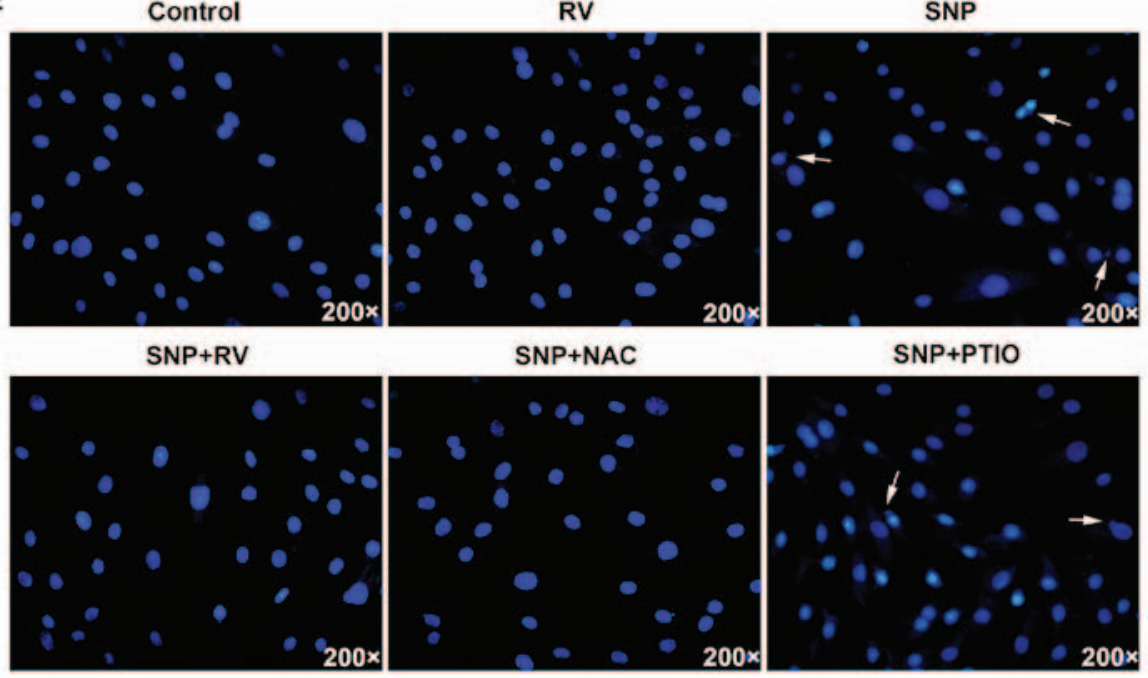

SNP+PTIO

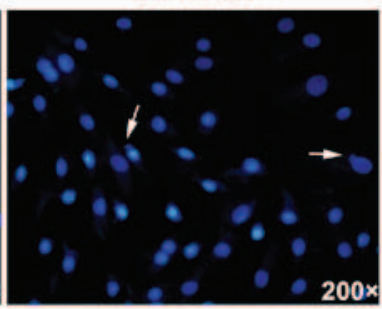

Figure 2. Effects of resveratrol (RV), $\mathrm{N}$-acetyl cysteine (NAC) and PTIO on sodium nitroprusside (SNP) induced nucleus pulposus (NP) cell apoptosis. (A) NP cell apoptosis analyzed by flow cytometry (FCM) with Annexin V/propidium iodide (PI) staining. (B) Quantitative analysis of apoptosis rates. ${ }^{* *} \mathrm{p}<0.01$, compared to control; ${ }^{\# \#} \mathrm{p}<0.01$, compared to SNP alone group. (C) Effects of RV, NAC and PTIO on SNP induced NP cell cytotoxiity analyzed by Cell Counting Kit-8 (CCK-8) assay. ${ }^{* *} \mathrm{p}<0.01$, compared to control; ${ }^{* \#} \mathrm{p}<0.01$, compared to SNP alone group. (D) Effects of RV, NAC, PTIO and SNP on caspases activity. ${ }^{* *} \mathrm{p}<0.01$, compared to control; ${ }^{\# \#} \mathrm{p}<0.01$, compared to SNP alone group. (E) Hoechst 33258 staining images of NP cells. Arrows show the apoptotic nuclei containing condensed or fragmented chromatin.

effectively scavenge intracellular ROS instead of NO, subsequently protecting NP cells from SNP induced apoptosis.

$R V$ protects NP cells from SNP induced disruption of cytoskeletal and morphological structure. Actin-Tracker Green and Tubulin-Trakcer Red were used to mark F-actin and $\alpha$-tubulin respectively under fluorescent microscope. In control and RV treated NP cells, normal cytoskeleton was observed with regularly distributed F-actin filament and microtubule (Fig. 4A). SNP treatment caused cytoskeleton shrinkage, by curling up F-actin filaments and disrupting microtubule structure (Fig. 4A). The SNP induced cytoskeleton shrinkage was significantly rescued by RV and NAC instead of PTIO (Fig. 4A). Under microscope with transmitted light illumination, SNP induced obvious cell shrinkage compared to control, which was significantly prevented by RV and NAC but not PTIO (Fig. 4B).

$R V$ protects against SNP induced NP cell apoptosis by scavenging ROS instead of NO ex vivo. Organ culture was also carried out to analyze effects of RV on NP cells ex vivo. Treatment of SNP ex vivo significantly increased the TUNEL-positive cells 


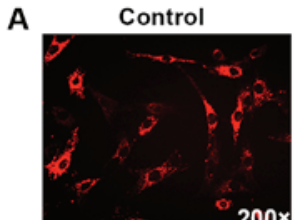

SNP+RV

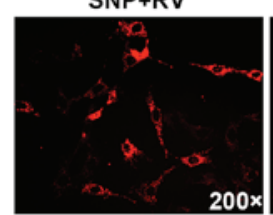

C
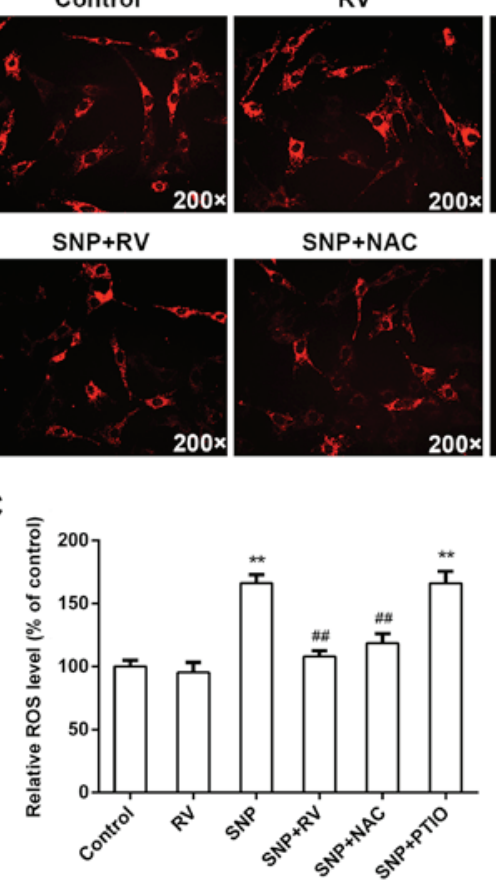

SNP+NAC

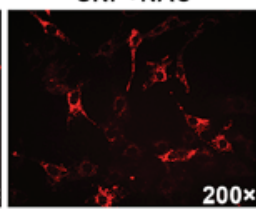

SNP

SNP+PTIO

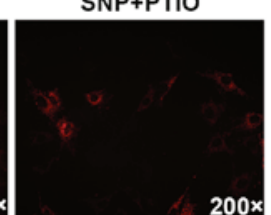

D

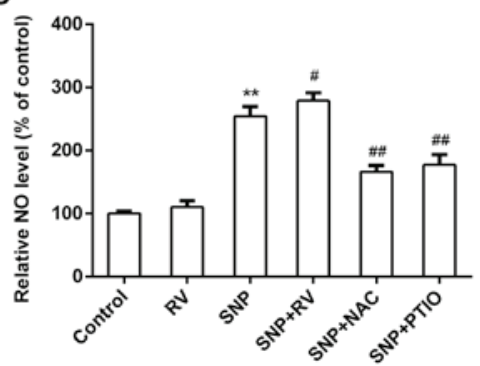

Figure 3. Mitochondrial membrane potential $(\Delta \Psi \mathrm{m})$, intracellular reactive oxygen species (ROS) and nitric oxide (NO) assay. (A) Tetramethylrhodamine methyl ester (TMRM) staining images of nucleus pulposus (NP) cells. (B) Quantitative analysis of TMRM signal intensity. TMRM signal intensity was expressed as mean density with IPP. ${ }^{* *} \mathrm{p}<0.01$, compared to control; ${ }^{\# \#} \mathrm{p}<0.01$, compared to sodium nitroprusside (SNP) alone group. (C) Relative intracellular ROS levels of different treatment. ${ }^{* *} \mathrm{p}<0.01$, compared to control; ${ }^{\# \#} \mathrm{p}<0.01$, compared to SNP alone group. (D) Relative intracellular NO levels of different treatment. ${ }^{* *} \mathrm{p}<0.01$, compared to control; ${ }^{\#} \mathrm{p}<0.05,{ }^{\# \#} \mathrm{p}<0.01$, compared to SNP alone group.

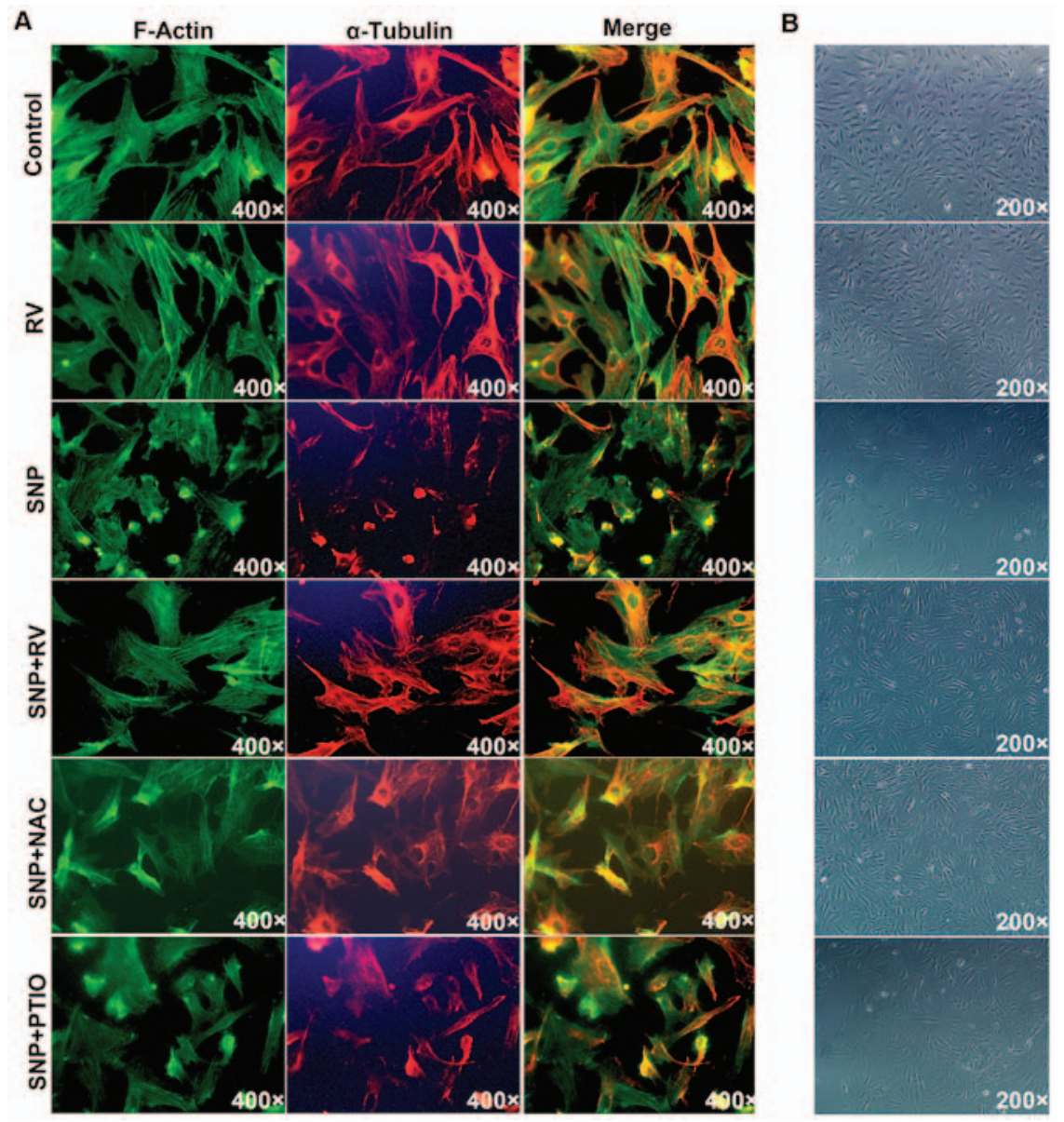

Figure 4. Effects of resveratrol (RV), N-acetyl cysteine (NAC) and PTIO on sodium nitroprusside (SNP) induced disruption of cytoskeletal and morphological structure of nucleus pulposus (NP) cells. (A) Actin-Tracker Green and Tubulin-Trakcer Red staining images of NP cells. (B) Morphological images of NP cells under transmitted light illumination using microscope. 

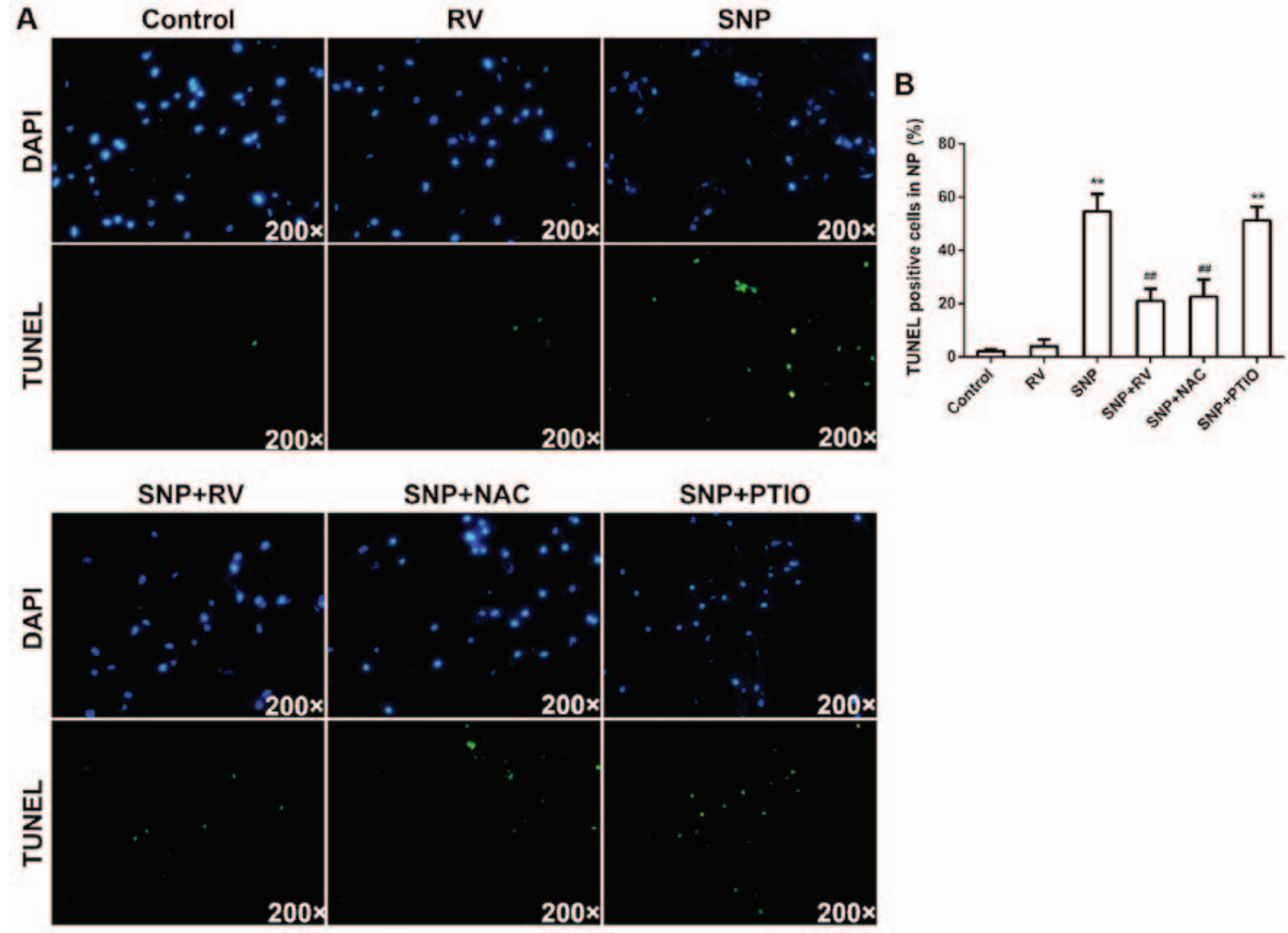

Figure 5. Effects of resveratrol (RV), N-acetyl cysteine (NAC) and PTIO on sodium nitroprusside (SNP) induced nucleus pulposus (NP) cell apoptosis ex vivo. (A) 4',6-Diamidino-2-phenylindole (DAPI) and TUNEL staining images of NP area on mid-sagittal sections of discs. (B) Quantitative analysis of TUNEL-positive cells percentage. ${ }^{* *} \mathrm{p}<0.01$, compared to control; ${ }^{\# \#} \mathrm{p}<0.01$, compared to SNP alone group.

percentage of NP area on mid-sagittal sections of discs compared to control (Fig. 5A and B). Consistent with in vitro results, $\mathrm{RV}$ and NAC significantly decreased TUNEL-positive cells percentage on mid-sagittal sections of discs compared to SNP treated group, while PTIO exerted no significant effects (Fig. 5A and B).

\section{Discussion}

Our results demonstrate that SNP can potently induce NP cell apoptosis in a ROS dependent rather than NO dependent manner. For the first time, RV is found to effectively scavenge ROS instead of NO in NP cells, through which RV significantly inhibits SNP induced NP cell apoptosis.

In previous cartilaginous studies, SNP is usually adopted as a $\mathrm{NO}$ donor to investigate $\mathrm{NO}$ related apoptosis and $\mathrm{NO}$ is considered to be the key mediator of apoptosis $(18,36,37)$. As to IVD, Zhao et al proved that both endoplasmic reticulum and mitochondria play a role in SNP induced annulus fibrosus cell apoptosis and speculated that high concentration of $\mathrm{NO}$ provided by SNP acted as the main upstream mediator (24). However, no study focused on effects of SNP on NP cells. Moreover, a recent study show that ROS rather than NO plays the key role in SNP induced in rabbit articular chondrocytes apoptosis, suggesting a new mechanism of SNP induced apoptosis (29). In our study, both intracellular NO and ROS level was raised by SNP treatment. Nevertheless, PTIO, which significantly scavenged intracellular NO, could not retard SNP induced NP cell cytotoxicity and apoptosis, which indicated that SNP induces $\mathrm{NP}$ cell apoptosis in a NO independent manner.
It has been reported that SNP can hinder electron transfer process and raise the level of reduced cytochrome $c$, resulting in production of ROS (38). Meanwhile, mitochondrial membrane potential can be reduced by SNP in many kinds of tissues (39-42). Loss of mitochondrial membrane potential means disruption of mitochondrial membrane function, which leads to the transfer of ROS into cytoplasm subsequently inducing cell death (43). As shown by our results, SNP induced an excessive production of ROS, loss of mitochondrial membrane potential and apoptosis. According to our results, we conclude that ROS instead of NO is the key mediator of SNP induced NP cell apoptosis. Interestingly, SNP seemed to exert no obvious effects on caspase- 8 activity when it significantly activated caspase- 3 and -9. It is probably due to that SNP induces NP cell apoptosis mainly via an intrinsic apoptotic pathway (44). In the present study, RV showed potent effects of scavenging ROS to inhibit SNP induced apoptosis, which is comparable to NAC. However, no obvious effects of RV were observed on SNP induced NO production unlike NAC and PTIO.

To further prove the effects of RV, an ex vivo study was also carried out. Among studies adopting disc organ culture system, few of them focus on NP cells apoptosis induced by oxidative stress (45-47). Considering the rapid effects of SNP in vitro, we modified the organ culture system with relatively short culture time. For the first time, it is found that ex vivo SNP treatment on IVD caused significant NP cell apoptosis under the established disc organ culture condition. Consistent with in vitro results, RV and NAC but not PTIO partly recued the NP cell apoptosis ex vivo. 
RV has been reported to exert antioxidative effects in many kinds of tissues, while the antioxidative potential of RV in NP cells is proved by us for the first time (48-50). As oxidative stress plays an important role in degeneration related disc cell apoptosis, antioxidative treatment is a promising therapeutic strategy (10-13). There have been studies aiming to retard IVD degeneration with antioxidative drugs and biomaterials $(13,51,52)$. The antioxidative potential makes $\mathrm{RV}$ a good choice for treatment of IVD degeneration in future targeting at degeneration related disc cell apoptosis.

In general, the present study demonstrated that ROS is the key mediator in SNP induced NP cell apoptosis. RV significantly inhibit SNP induced NP cell apoptosis by scavenging ROS but not NO in vitro and ex vivo. With potent antioxidative activity, RV would be a favorable candidate for protection against oxidative stress related disc cell apoptosis.

\section{Acknowledgements}

The technical support from Chuan Jiang and Lei Wang is appreciated by the authors.

\section{Competing interests}

The authors declare that they have no competing interests.

\section{References}

1. Hart LG, Deyo RA and Cherkin DC: Physician office visits for low back pain. Frequency, clinical evaluation, and treatment patterns from a U.S. national survey. Spine 20: 11-19, 1995.

2. Katz JN: Lumbar disc disorders and low-back pain: socioeconomic factors and consequences. J Bone Joint Surg Am 88 (Suppl 2): 21-24, 2006.

3. Schwarzer AC, Aprill CN, Derby R, Fortin J, Kine G and Bogduk N: The relative contributions of the disc and zygapophyseal joint in chronic low back pain. Spine 19: 801-806, 1994.

4. Dario AB, Ferreira ML, Refshauge KM, Lima TS, Ordoñana JR and Ferreira PH: The relationship between obesity, low back pain, and lumbar disc degeneration when genetics and the environment are considered: a systematic review of twin studies. Spine J 15: 1106-1117, 2015.

5. Walsh AJ and Lotz JC: Biological response of the intervertebral disc to dynamic loading. J Biomech 37: 329-337, 2004.

6. Ahsan R, Tajima N, Chosa E, Sugamata M, Sumida M and Hamada M: Biochemical and morphological changes in herniated human intervertebral disc. J Orthop Sci 6: 510-518, 2001.

7. Ding F, Shao ZW and Xiong LM: Cell death in intervertebral disc degeneration. Apoptosis 18: 777-785, 2013.

8. Kepler CK, Ponnappan RK, Tannoury CA, Risbud MV and Anderson DG: The molecular basis of intervertebral disc degeneration. Spine J 13: 318-330, 2013.

9. Kowaltowski AJ and Vercesi AE: Mitochondrial damage induced by conditions of oxidative stress. Free Radic Biol Med 26 463-471, 1999.

10. Ding F, Shao ZW, Yang SH, Wu Q, Gao F and Xiong LM: Role of mitochondrial pathway in compression-induced apoptosis of nucleus pulposus cells. Apoptosis 17: 579-590, 2012.

11. Ma KG, Shao ZW, Yang SH, Wang J, Wang BC, Xiong LM, Wu Q and Chen SF: Autophagy is activated in compression-induced cell degeneration and is mediated by reactive oxygen species in nucleus pulposus cells exposed to compression. Osteoarthritis Cartilage 21: 2030-2038, 2013.

12. Park EY and Park JB: High glucose-induced oxidative stress promotes autophagy through mitochondrial damage in rat notochordal cells. Int Orthop 37: 2507-2514, 2013.

13. Yang L, Rong Z, Zeng M, Cao Y, Gong X, Lin L, Chen Y, Cao W, Zhu L and Dong W: Pyrroloquinoline quinone protects nucleus pulposus cells from hydrogen peroxide-induced apoptosis by inhibiting the mitochondria-mediated pathway. Eur Spine J 24: 1702-1710, 2015.
14. Kohyama K, Saura R, Doita M and Mizuno K: Intervertebral disc cell apoptosis by nitric oxide: biological understanding of intervertebral disc degeneration. Kobe J Med Sci 46: 283-295, 2000.

15. Fermor B, Christensen SE, Youn I, Cernanec JM, Davies CM and Weinberg JB: Oxygen, nitric oxide and articular cartilage. Eur Cell Mater 13: 56-65, discussion 65, 2007.

16. Studer RK, Gilbertson LG, Georgescu H, Sowa G, Vo N and Kang JD: p38 MAPK inhibition modulates rabbit nucleus pulposus cell response to IL-1. J Orthop Res 26: 991-998, 2008.

17. Katsuno R, Hasegawa T, Iwashina T, Sakai D, Mikawa Y and Mochida J: Age-related effects of cocultured rat nucleus pulposus cells and macrophages on nitric oxide production and cytokine imbalance. Spine 33: 845-849, 2008.

18. Blanco FJ, Ochs RL, Schwarz H and Lotz M: Chondrocyte apoptosis induced by nitric oxide. Am J Pathol 146: 75-85, 1995.

19. Yoon JB, Kim SJ, Hwang SG, Chang S, Kang SS and Chun JS: Non-steroidal anti-inflammatory drugs inhibit nitric oxide-induced apoptosis and dedifferentiation of articular chondrocytes independent of cyclooxygenase activity. J Biol Chem 278: 15319-15325, 2003.

20. Kim JS, Park ZY, Yoo YJ, Yu SS and Chun JS: p38 kinase mediates nitric oxide-induced apoptosis of chondrocytes through the inhibition of protein kinase $\mathrm{C}$ zeta by blocking autophosphorylation. Cell Death Differ 12: 201-212, 2005.

21. Wu GJ, Chen TG, Chang HC, Chiu WT, Chang CC and Chen RM: Nitric oxide from both exogenous and endogenous sources activates mitochondria-dependent events and induces insults to human chondrocytes. J Cell Biochem 101: 1520-1531, 2007.

22. Maneiro E, López-Armada MJ, de Andres MC, Caramés B, Martín MA, Bonilla A, Del Hoyo P, Galdo F, Arenas J and Blanco FJ: Effect of nitric oxide on mitochondrial respiratory activity of human articular chondrocytes. Ann Rheum Dis 64: 388-395, 2005.

23. Lee SW, Song YS, Shin SH, Kim KT, Park YC, Park BS, Yun I, Kim K, Lee SY, Chung WT, et al: Cilostazol protects rat chondrocytes against nitric oxide-induced apoptosis in vitro and prevents cartilage destruction in a rat model of osteoarthritis. Arthritis Rheum 58: 790-800, 2008.

24. Zhao CQ, Zhang YH, Jiang SD, Jiang LS and Dai LY: Both endoplasmic reticulum and mitochondria are involved in disc cell apoptosis and intervertebral disc degeneration in rats. Age (Dordr) 32: 161-177, 2010.

25. Haider UG, Sorescu D, Griendling KK, Vollmar AM and Dirsch VM: Resveratrol suppresses angiotensin II-induced Akt/protein kinase B and p70 S6 kinase phosphorylation and subsequent hypertrophy in rat aortic smooth muscle cells. Mol Pharmacol 62: 772-777, 2002.

26. Bhat KPL, Kosmeder JW 2nd and Pezzuto JM: Biological effects of resveratrol. Antioxid Redox Signal 3: 1041-1064, 2001.

27. Bertelli AA, Ferrara F, Diana G, Fulgenzi A, Corsi M, Ponti W, Ferrero ME and Bertelli A: Resveratrol, a natural stilbene in grapes and wine, enhances intraphagocytosis in human promonocytes: a co-factor in antiinflammatory and anticancer chemopreventive activity. Int J Tissue React 21: 93-104, 1999.

28. Jang M, Cai L, Udeani GO, Slowing KV, Thomas CF, Beecher CW, Fong HH, Farnsworth NR, Kinghorn AD, Mehta RG, et al: Cancer chemopreventive activity of resveratrol, a natural product derived from grapes. Science 275: 218-220, 1997.

29. Liang Q, Wang XP and Chen TS: Resveratrol protects rabbit articular chondrocyte against sodium nitroprusside-induced apoptosis via scavenging ROS. Apoptosis 19: 1354-1363, 2014.

30. Li X, Phillips FM, An HS, Ellman M, Thonar EJ, Wu W, Park D and Im HJ: The action of resveratrol, a phytoestrogen found in grapes, on the intervertebral disc. Spine 33: 2586-2595, 2008

31. Wuertz K, Quero L, Sekiguchi M, Klawitter M, Nerlich A, Konno S, Kikuchi S and Boos N: The red wine polyphenol resveratrol shows promising potential for the treatment of nucleus pulposus-mediated pain in vitro and in vivo. Spine 36: E1373-E1384, 2011.

32. Wang D, Hu Z, Hao J, He B, Gan Q, Zhong X, Zhang X, Shen J, Fang $\mathrm{J}$ and Jiang W: SIRT1 inhibits apoptosis of degenerative human disc nucleus pulposus cells through activation of Akt pathway. Age (Dordr) 35: 1741-1753, 2013.

33. Jiang W, Zhang X, Hao J, Shen J, Fang J, Dong W, Wang D, Zhang X, Shui W, Luo Y, et al: SIRT1 protects against apoptosis by promoting autophagy in degenerative human disc nucleus pulposus cells. Sci Rep 4: 7456, 2014. 
34. Xia X, Guo J, Lu F and Jiang J: SIRT1 plays a protective role in intervertebral disc degeneration in a puncture-induced Rodent model. Spine 40: E515-E524, 2015.

35. Li K, Li Y, MaZ and Zhao J: Crocin exerts anti-inflammatory and anti-catabolic effects on rat intervertebral discs by suppressing the activation of JNK. Int J Mol Med 36: 1291-1299, 2015.

36. Del Carlo M Jr and Loeser RF: Nitric oxide-mediated chondrocyte cell death requires the generation of additional reactive oxygen species. Arthritis Rheum 46: 394-403, 2002.

37. Eo SH, Cho H and Kim SJ: Resveratrol inhibits nitric oxideinduced apoptosis via the NF-kappa B pathway in rabbit articular chondrocytes. Biomol Ther (Seoul) 21: 364-370, 2013.

38. Giorgio M, Migliaccio E, Orsini F, Paolucci D, Moroni M, Contursi C, Pelliccia G, Luzi L, Minucci S, Marcaccio M, et al: Electron transfer between cytochrome $c$ and $\mathrm{p} 66^{\text {Shc }}$ generates reactive oxygen species that trigger mitochondrial apoptosis. Cell 122: 221-233, 2005.

39. Wang CN, Duan GL, Liu YJ, Yu Q, Tang XL, Zhao W, Li XH, Zhu XY and Ni X: Overproduction of nitric oxide by endothelial cells and macrophages contributes to mitochondrial oxidative stress in adrenocortical cells and adrenal insufficiency during endotoxemia. Free Radic Biol Med 83: 31-40, 2015.

40. Baek MW, Seong KJ, Jeong YJ, Kim GM, Park HJ, Kim SH, Chung HJ, Kim WJ and Jung JY: Nitric oxide induces apoptosis in human gingival fibroblast through mitochondria-dependent pathway and JNK activation. Int Endod J 48: 287-297, 2015.

41. de Andrés MC, Maneiro E, Martín MA, Arenas J and Blanco FJ: Nitric oxide compounds have different effects profiles on human articular chondrocyte metabolism. Arthritis Res Ther 15: R115, 2013.

42. Mahesh R, Jung HW, Kim GW, Kim YS and Park YK: Cryptotanshinone from Salviae miltiorrhizae radix inhibits sodium-nitroprusside-induced apoptosis in neuro-2a cells. Phytother Res 26: 1211-1219, 2012.

43. Fleury C, Mignotte B and Vayssière JL: Mitochondrial reactive oxygen species in cell death signaling. Biochimie 84 : 131-141, 2002

44. Ryter SW, Kim HP, Hoetzel A, Park JW, Nakahira K, Wang X and Choi AM: Mechanisms of cell death in oxidative stress. Antioxid Redox Signal 9: 49-89, 2007.

45. Markova DZ, Kepler CK, Addya S, Murray HB, Vaccaro AR, Shapiro IM, Anderson DG, Albert TJ and Risbud MV: An organ culture system to model early degenerative changes of the intervertebral disc II: profiling global gene expression changes. Arthritis Res Ther 15: R121, 2013.
46. Kim JS, Ellman MB, Yan D, An HS, Kc R, Li X, Chen D, Xiao G, Cs-Szabo G, Hoskin DW, et al: Lactoferricin mediates anti-inflammatory and anti-catabolic effects via inhibition of IL-1 and LPS activity in the intervertebral disc. J Cell Physiol 228: 1884-1896, 2013.

47. Risbud MV, Di Martino A, Guttapalli A, Seghatoleslami R, Denaro V, Vaccaro AR, Albert TJ and Shapiro IM: Toward an optimum system for intervertebral disc organ culture: TGF-beta 3 enhances nucleus pulposus and anulus fibrosus survival and function through modulation of TGF-beta-R expression and ERK signaling. Spine 31: 884-890, 2006.

48. Wu H, Li GN, Xie J, Li R, Chen QH, Chen JZ, Wei ZH, Kang LN and $\mathrm{Xu} \mathrm{B}$ : Resveratrol ameliorates myocardial fibrosis by inhibiting ROS/ERK/TGF- $\beta$ /periostin pathway in STZ-induced diabetic mice. BMC Cardiovasc Disord 16: 5, 2016.

49. Abengózar-Vela A, Calonge M, Stern ME, González-García MJ and Enríquez-De-Salamanca A: Quercetin and resveratrol decrease the inflammatory and oxidative responses in human ocular surface epithelial cells. Invest Ophthalmol Vis Sci 56: 2709-2719, 2015.

50. Wang R, Liu YY, Liu XY, Jia SW, Zhao J, Cui D and Wang L: Resveratrol protects neurons and the myocardium by reducing oxidative stress and ameliorating mitochondria damage in a cerebral ischemia rat model. Cell Physiol Biochem 34: 854-864, 2014

51. Yang X, Jin L, Yao L, Shen FH, Shimer AL and Li X: Antioxidative nanofullerol prevents intervertebral disk degeneration. Int J Nanomedicine 9: 2419-2430, 2014.

52. Cheng YH, Yang $\mathrm{SH}$ and Lin FH: Thermosensitive chitosan-gelatin-glycerol phosphate hydrogel as a controlled release system of ferulic acid for nucleus pulposus regeneration. Biomaterials 32: 6953-6961, 2011.

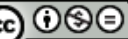

This work is licensed under a Creative Commons Attribution-NonCommercial-NoDerivatives 4.0 International (CC BY-NC-ND 4.0) License. 